\title{
Semi-Automatic Noninvasive Tracking of Local Myocardial Motion using Dynamic Programming based Elastic Model
}

\author{
Chen Chao \\ Mie University \\ Kurimamachiya-cho \\ 1577 , \\ Tsu, Mie 514-8507, \\ Japan
}

\author{
Wataru Ohyama \\ Mie University \\ Kurimamachiya-cho \\ 1577 , \\ Tsu, Mie 514-8507, \\ Japan
}

\author{
Tetsushi \\ Wakabayashi \\ Mie University \\ Kurimamachiya-cho \\ 1577 , \\ Tsu, Mie 514-8507, \\ Japan
}

\author{
Fumitaka Kimura \\ Mie University \\ Kurimamachiya-cho \\ 1577, \\ Tsu, Mie 514-8507, \\ Japan
}

\begin{abstract}
A semi-automatic motion-tracking method for local myocardial tissue on M-mode echocardiograms is proposed in this paper. The proposed method is applicable to estimating myocardial performance in clinics. The M-mode echocardiogram is a type of echocardiogram used in clinics to measure diagnostic indexes. Such as the thickening and thinning of myocardial muscle layers. In order to measure such indexes, doctors are required to manually track myocardial motion. However, tracking myocardial motion by hand is a very tedious and time-consuming process. The proposed method for tracking the motion of myocardial tissue is based on Dynamic Programming (DP). A Myocardial Elastic (ME) Model is employed to reduce the accumulation of velocity error. The experiment has 3 parts: visual inspection, statistical estimation and the analysis of systematic error. The results of these three evaluations indicate that the proposed method can provide more accurate motion tracking and can replace the manual tracking method for doctors in clinics.
\end{abstract}

\section{General Term}

Medical Image Processing, Biomedical Analysis, Signal Processing

\section{Keywords}

Myocardial Motion Tracking, Ultrasonic Imaging, M-mode Echocardiogram, Dynamic Programming, Elastic Model

\section{INTRODUCTION}

Echocardiography is widely employed as an essential and necessary modality for the diagnosis and treatment of cardiovascular diseases. The use of echocardiograms in clinical cardiology provides multi-fold advantages, including noninvasiveness of diagnosis and real-time imaging. In clinical cardiology, diagnosis and especially analysis rely on visual inspection or manual measurements by experienced cardiologists. Manual methods are tedious and time consuming, and visual assessment tends toward qualitative and subjective diagnoses that suffer from considerable interand intra-observer variability. Therefore, an automated computer-based analysis is highly desirable to obtain more objective and quantitative diagnoses.

Figure 1 shows an example of clinical echocardiograms. In the figure, (a) and (b) show B-mode and M-mode echocardiograms, respectively. The M-mode echocardiogram is widely used in clinics to measure diagnostic indexes such as the thickening and thinning of myocardial muscle layers. It is a diagnostic ultrasonic presentation in which the distance of echo-producing interfaces from a transducer is displayed along the vertical axis and time is displayed along the horizontal axis. The recording motion of the myocardium is towards and away from the transducer. In early-stage of myocardial diseases, such as amyloidosis and hypertrophic cardiomyopathy, affection starts to appear on small tissue in regional myocardium. The automatic analysis is also expected to determine these early-stage affections.

Several approaches have been proposed to quantify the motion of the myocardium from two-dimensional (2-D) echocardiograms [1]-[3]. However, in these approaches, the spatial and temporal resolutions of quantification and tracking of myocardial motion strongly depend on those of imaging performance of ultrasonic equipment. In particularly, echocardiograms employ low-pass filters on ultrasonic signals in the image generation process. Consequently, the spatial resolution of images decreases and is insufficient for detailed diagnosis of local, inner myocardial tissues. To enable quantitative evaluation of a tissue's kinetic performance on inner myocardial tissues, accurate and suitable tracking of the myocardium with high spatial resolution is required. To overcome the problem of low temporal resolution, several approaches employ raw ultrasonic signals from which echocardiograms are generated [4]-[7].

In this context, the authors have proposed myocardial motiontracking methods employing M-mode ultrasonic radio frequency (RF) and Doppler signals. The first attempts at automatic tracking of a regional myocardium employed Mmode ultrasonic echoes of an RF signal [8]. As only spatial distribution of ultrasonic echoes could be obtained, this tracking method utilized a correlation-based block-matching method to handle noisy ultrasonic signals. The authors also proposed a motion-tracking method using ultrasonic pulsed Doppler, which is a modality that provides the instantaneous velocity of tissues [9]. In this method, the authors utilized a correlation-weighted mean filter and tracking stabilization with a myocardial elastic model to suppress noise effects in 


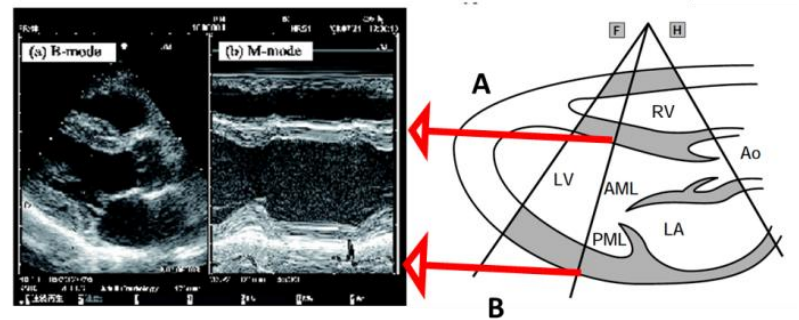

Figure 1: Example of clinical echocardiograms: (a) Bmode and (b) M-mode echocardiograms: A. interventricular septum and $B$. left-ventricular posterior wall.

the velocity calculation. Myocardial motion tracking using a Dynamic Programming (DP) tracking approach is another method for stable motion tracking [10]. The authors confirmed that global optimization of evaluation function using DP provides more accurate and stable tracking results than that found by the accumulation of instantaneous velocity. This paper introduces tracking stabilization using a Myocardial Elastic (ME) model for a DP-based tracking method. This article is a comprehensive extension of our previous brief reports [11]-[12].

The proposed approach employs an M-mode ultrasonic Doppler signal for velocity estimation and a DP-based tracking method. DP is a method for solving problems that exhibiting the properties of overlapping sub-problems and optimal substructure. In this method, DP is employed to find a trajectory that minimizes the objective function. The MEmodel is employed to provide more accurate motion tracking throughout, by modifying the distance between tracking points.

\section{MYOCARDIAL MOTION TRACKING}

Here, the authors describe how to track the local myocardial motion using the proposed DP with an ME-model tracking method. The method consists of two main stages, as shown in Figure 2. In the first stage, the velocity field in the myocardium is estimated and the correlation-weighted mean filter was applied to suppress noise effects [10]. Next, in the motion-tracking stage, the instantaneous displacement of a set of tracking points is calculated from the estimated velocity field and the DP tracking method with the ME-model tracks the motion of the tracking points.

\subsection{Acquisition system for M-mode ultrasonic Doppler signal}

To assess inner-wall myocardial performance, myocardial motion should be tracked with high spatial resolution. For instance, a normal left-ventricular myocardium has a thickness of 10 millimeters, and so spatial resolution on the order of sub-millimeters is required. The authors employed an M-mode ultrasonic Doppler signal to achieve motion tracking with such high spatial resolution. Figure 3 illustrates the block-diagram of the signal acquisition system used in this research.

The details of the acquisition system are described in [10]. We obtain a discrete Doppler signal $Z=\left\{z\left(x_{i}, t_{k}\right)\right\}$ consisting of a sine component $z_{s}\left(x_{i}, t_{k}\right)$ and a cosine component $z_{c}\left(x_{i}, t_{k}\right)$ represented by a complex expression as follows:

$$
z\left(x_{i}, t_{k}\right)=z_{s}\left(x_{i}, t_{k}\right)+j z_{c}\left(x_{i}, t_{k}\right),
$$

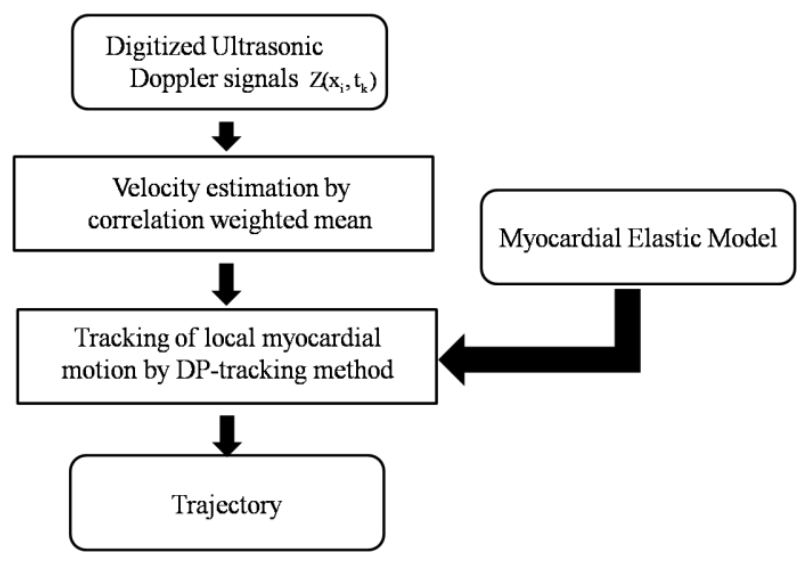

Figure 2: Flow chart of the myocardial motion tracking process.

$$
\begin{aligned}
& x_{i}=\frac{i c_{0} T_{s}}{2}, \\
& t_{k}=k \Delta T,
\end{aligned}
$$

where $x_{i}$ and $t_{k}$ are the distance from the ultrasonic transducer to $i$-th sampling point and the time when the $k$-th ultrasonic plus is transmitted, respectively. The constant $c_{0}$ is the speed of sound in a human body, i.e., $1530[\mathrm{~m} / \mathrm{s}] . T_{s}$ and $\Delta T$ are the sampling period and repetition period of the ultrasonic pulse, respectively, and $j$ is the imaginary unit.

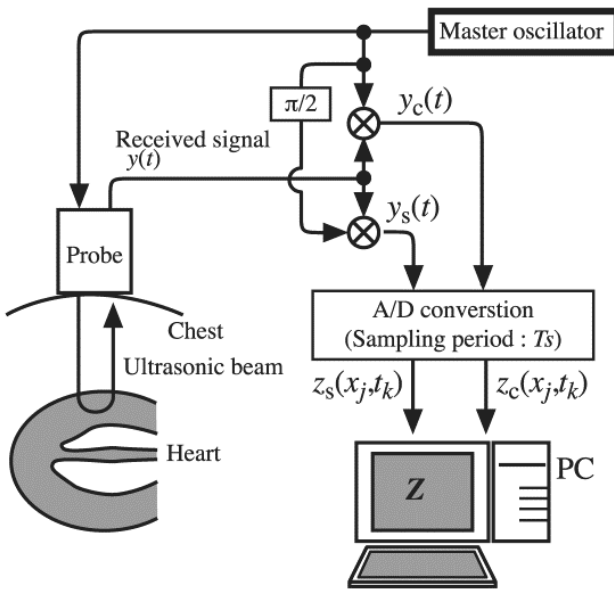

Figure 3: Block-diagram of the data acquisition system.

\subsection{Velocity estimation for myocardium}

The instantaneous velocity of each sampling point $v\left(x_{j}, t_{k}\right)$ is first derived from the discrete Doppler signal in the same way used in our former method [10].

The proposed method reduces velocity estimation errors due to noise and speckling by taking the correlation-weighted mean of the velocities at several sampling points. The authors obtain the correlation-weighted mean velocity $v^{\prime}\left(x_{j}, t_{k}\right)$ at a tracking point $x_{j}$.

The detailed calculation process for $v\left(x_{i}, t_{k}\right)$ and $v^{\prime}\left(x_{i}, t_{k}\right)$ is described in [9][10]. 


\subsection{DP tracking method}

In this research, the authors formulate local myocardial motion using an M-mode ultrasonic Doppler signal as followings. First, the authors define an M-mode ultrasonic Doppler signal that is acquired from a subject as,

$$
\begin{gathered}
Z=\left\{z\left(x_{i}, t_{k}\right)\right\}, \text { for } \\
i=0,1,2, \ldots, N-1, k=0,1,2, \ldots, L-1,
\end{gathered}
$$

where $N$ and $L$ denote the number of sampling points along the ultrasonic beam and the number of ultrasonic beams contained in one data acquisition, respectively. To simplify the following notation, a set of sampling points at time $t_{k}$ is denoted by:

$$
x_{k}=\left\{x_{i} \mid t_{k}\right\}
$$

Of course, $x_{k}=x_{k+1}$ for all $k$.

Next, a starting point for tracking is located at the depth that corresponds to the location of the myocardium at the tracking start time $t_{0}$. We express the tracking point $d_{0}$ as equivalent to that of one of the sampling points $x_{0}$.

$$
d_{0}=x_{i} \mid x_{i} \in x_{0}
$$

Similarly, a tracking point at timing $t_{k}$ is denoted by:

$$
d_{k}=x_{i} \mid x_{i} \in x_{k} .
$$

Using the above notation, the motion tracking process of which the start point $d_{0}$, is equivalent to determining a set of tracking points:

$$
\begin{gathered}
D=\left(d_{0}, d_{1}, d_{2}, \ldots, d_{L-1}\right), \text { where } \\
d_{k}=x_{i} \mid x_{i} \in x_{k} .
\end{gathered}
$$

When multiple tracking points are employed in tracking, tracking points at $t_{k}$ are expressed as a multiplet $\boldsymbol{d}_{k}$ :

$$
\boldsymbol{d}_{k}=\left(d_{k}^{(0)}, d_{k}^{(1)} d_{k}^{(2)}, \ldots, d_{k}^{(M-1)}\right)
$$

where $M$ denotes the number of tracking points.

The authors consider that the motion tracking process is equivalent to determining a trajectory $\boldsymbol{D}$, which minimizes the objective function:

$$
f(\boldsymbol{D})=\sum_{k=1}^{L-1}\left\{d_{k-1}+\Delta T v^{\prime}\left(d_{k-1}, t_{k-1}\right)-d_{k}\right\}^{2},
$$

under the assumption that the start and end points $d_{0}, d_{L-1}$ are equal to each other.

The trajectory $\boldsymbol{D}$ that minimizes Eq. (9) can be determined using the DP algorithm described as followings. The partial sum of Eq. (9) on $D_{l}=\left(d_{0}, d_{1}, d_{2}, \ldots, d_{l-1}\right),(l<L)$ is defined by:

$$
f\left(\boldsymbol{D}_{l}\right)=\sum_{k=1}^{l-1}\left\{d_{k-1}+\Delta T v^{\prime}\left(d_{k-1}, t_{k-1}\right)-d_{k}\right\}^{2} .
$$

When the position of tracking point $d_{k}$ at $t_{l}$ is equal to $x^{(l)}$, the authors can rewrite Eq. (10):

$$
\begin{gathered}
f\left(\boldsymbol{D}_{l} ; x^{(l)}\right)=\left\{d_{l-1}+\Delta T v^{\prime}\left(d_{l-1}, t_{l-1}\right)-x^{(l)}\right\}^{2}+ \\
\sum_{k=1}^{l-2}\left\{d_{k-1}+\Delta T v^{\prime}\left(d_{k-1}, t_{k-1}\right)-d_{k}\right\}^{2} .
\end{gathered}
$$

The minimum of $f\left(D_{l} ; x^{(l)}\right)$ is expressed by the following recurrence equation:

$$
\begin{aligned}
f_{\min }\left(\boldsymbol{D}_{l} ; x^{(l)}\right) & \\
& =\min _{x^{(l-1)}}\left[\left\{x^{(l-1)}+\Delta T v^{\prime}\left(x^{(l-1)}, t_{l-1}\right)-x^{(l)}\right\}^{2}\right. \\
& \left.+f_{\min }\left(\boldsymbol{D}_{(l-1)} ; x^{(l-1)}\right)\right],
\end{aligned}
$$

The authors can effectively calculate the minimum of Eq. (9) as $f\left(\boldsymbol{D}_{L-1} ; d_{0}\right)$ from Eq. (12) using DP, and $\left(\boldsymbol{D}_{L-1} ; d_{0}\right)$ is obtained as the optimal trajectory of a tracking point located on the regional myocardium.

\subsection{DP Tracking Method with Myocardial Elastic Model}

Although the authors can achieve stable motion tracking under the assumption that the start and end points are equal to each other using the DP tracking method, the occurrence of extreme motion tracking error cannot be avoided. Since the motion tracking with the DP method is an independent process corresponding to each tracking point, there is no any mutual action between two tracking points. The mutual action can avoid extreme tracking error throughout adjusting mutual distance between tracking points. This paper introduces the ME-model to modify the error and the interaction by limiting the distance between tracking points.

The position of the $M$ tracking points at time $t_{k+1}$ is expressed by:

$$
\boldsymbol{d}_{k+1}=\boldsymbol{d}_{k}+\Delta T \boldsymbol{v}_{k},
$$

where $\boldsymbol{d}_{k}$ and $\boldsymbol{d}_{k+1}$ are expressed as a multiplet defined by Eq. (8). We introduce a correction vector $\boldsymbol{\varepsilon}_{k}$ to the positions of the tracking points as follows:

$$
\begin{gathered}
\boldsymbol{d}_{k+1}^{\prime}=\boldsymbol{d}_{k+1}+\boldsymbol{\varepsilon}_{k} \\
=\boldsymbol{d}_{k}+\Delta T \boldsymbol{v}_{k}+\boldsymbol{\varepsilon}_{k}, \text { where } \\
\boldsymbol{\varepsilon}_{k}=\left(\boldsymbol{\varepsilon}_{k}^{(0)}, \boldsymbol{\varepsilon}_{k}^{(1)}, \varepsilon_{k}^{(2)}, \ldots \varepsilon_{k}^{(M-1)}\right) .
\end{gathered}
$$

The correction vector is one that minimizes an objective function defined by:

$$
\begin{aligned}
\boldsymbol{e}\left(\boldsymbol{\varepsilon}_{k}\right)= & \sum_{i=0}^{M-1} \varepsilon_{k}^{(i)^{2}} \\
& +\frac{E}{2} \sum_{i=0}^{M-2}\left\{\left(\boldsymbol{d}_{k+1}^{(i+1)^{\prime}}-\boldsymbol{d}_{k+1}^{(i)^{\prime}}\right)-\left(\boldsymbol{d}_{0}^{i+1}-\boldsymbol{d}_{0}^{i}\right)\right\}^{2},
\end{aligned}
$$

where $\boldsymbol{d}_{0}$ and $E$ are an initial position vector and a parameter of elasticity, respectively. If $E$ is zero, then $\boldsymbol{\varepsilon}_{k}$ is also a zero vector (i.e., no correction is performed). As the value of $E$ increases, the distances between tracking points $\boldsymbol{d}_{0}$ tend to be unchanged. 
To utilize the myocardial elastic model with DP-tracking, we adopted a modification to the original myocardial elastic model. DP is employed to realize motion tracking only on the $l$-th tracking point using Eq. (10).

$$
\begin{gathered}
\boldsymbol{e}\left(\boldsymbol{\varepsilon}_{k}\right)=\boldsymbol{e}\left(\boldsymbol{\varepsilon}_{\boldsymbol{k}}^{(\boldsymbol{U})}\right)+\boldsymbol{e}\left(\boldsymbol{\varepsilon}_{k}^{(L)}\right), \\
\boldsymbol{e}\left(\boldsymbol{\varepsilon}_{k}^{(\boldsymbol{U})}\right)=\sum_{i=0}^{l-1} \boldsymbol{\varepsilon}_{k}^{(i)^{2}} \\
+\frac{E}{2} \sum_{j=\mathbf{0}}^{l-2}\left\{\left(d_{k+1}^{(j+1)}-d_{k+1}^{(j)}\right)+\left(d_{0}^{(j+1)}-d_{0}^{(j)}\right)\right\}^{2}, \\
\boldsymbol{e}\left(\boldsymbol{\varepsilon}_{\boldsymbol{k}}^{(L)}\right)=\sum_{i=l+1}^{M-1} \boldsymbol{\varepsilon}_{k}^{(i)^{2}} \\
+\frac{E}{2} \sum_{j=l}^{M-2}\left\{\left(d_{k+1}^{\prime(j+1)}-d_{k+1}^{(j)}\right)+\left(d_{0}^{j+1}-d_{0}^{(j)}\right)\right\}^{2} .
\end{gathered}
$$

In Eqs.(18) and (19), the depth of the $l$-th tracking point is defined under the assumption that $\boldsymbol{d}_{k+1}^{\prime(i)}=\boldsymbol{d}_{k+1}^{(i)}$. In this study, $l$ is set to 3 and $M$ is set to 10 . This means that the DP method is used to track the motion of the third point.

\section{EXPERIMENT AND RESULTS}

\subsection{Experiment}

To confirm the tracking performance of the proposed DP with an ME-model tracking method, the authors conducted an experiment in which clinical ultrasonic signals were used for motion tracking. The authors made a visual comparison of the tracking results found from using Simple Tracking, Correlation Weighted Phase-difference (CWP) [10], and the proposed DP with the ME-model.

M-mode ultrasonic Doppler signals were acquired using ultrasonic diagnostic equipment (Hitachi, EUB 6500 Advanced). There were 46 acquired ultrasonic signals: 23 targeted the inter-ventricular septum (IVS, Figure 1A) and 23 targeted the left-ventricular posterior wall (LVPW, Figure 1B), respectively. The starting points for motion tracking were manually determined on the myocardial tissues by a trained medical image researcher for each ultrasonic signal.

One cardiac cycle was detected accurately using an $\mathrm{R}$ wave to $R$ wave (R-R) time interval on an electrocardiogram (ECG) obtained in synchronization with the ultrasonic signal. Therefore, each tracking point is expected to return to the same depth position in depth as at the commencement of tracking. In this experiment, the authors obtained the optimal result for $E=0.03$.

The authors employed the difference between two trajectories obtained by automatic and manual tracking as a measure of tracking accuracy. An operator first traced the motion of the endocardium (inside of the heart) and epicardium (outside of heart). The two automatic tracking methods were also applied to trace the motion of the local myocardium in the same region as manual tracking. The mean difference in trajectory $D$ is defined by:

$$
\begin{gathered}
\boldsymbol{e}\left(\boldsymbol{m}_{k}, \boldsymbol{a}_{k}\right)=\sqrt{\frac{\sum_{k=0}^{M-1}\left(\left(\boldsymbol{m}_{k}, \boldsymbol{a}_{k}\right)^{\boldsymbol{T}}\left(\boldsymbol{m}_{k}-\boldsymbol{a}_{k}\right)\right)}{M}} \\
D=\frac{\sum_{k=0}^{L-1} \boldsymbol{e}\left(\boldsymbol{m}_{k}, \boldsymbol{a}_{k}\right)}{L}
\end{gathered}
$$

where $\boldsymbol{m}_{k}=\left(m_{k}^{(0)}, m_{k}^{(1)}, m_{k}^{(2)}, \ldots, m_{k}^{(M-1)}\right)$ and $\boldsymbol{a}_{k}=$ $\left(a_{k}^{(0)}, a_{k}^{(1)}, a_{k}^{(2)}, \ldots, a_{k}^{(M-1)}\right)$ are tracking points at time $t_{k}$ calculated by manual tracking and automatic tracking, respectively. The number of tracking points $M$ is 10 . Let $L$ defined as the number of pulse repetitions in one heart beat cycle.

In our study, we define the mean error as:

$$
M_{D}=\frac{\sum_{n=1}^{T} D_{n}}{T}
$$

where $D_{n}$ is the difference between two trajectories for the $n$ th subject.

In this study, $T$ is the number of examples. The authors used 46 examples, in which 23 subjects were for the IVS and the other 23 were for the LVPW.

The standard deviation of the difference is defined as:

$$
\boldsymbol{\sigma}_{D}=\sqrt{\frac{1}{T} \sum_{n=1}^{T}\left(\boldsymbol{D}_{\boldsymbol{n}}-\boldsymbol{M}_{\boldsymbol{D}}\right)^{2}},
$$

where $T$ is also defined as the number of examples.

\subsection{Results and Discussion}

The trajectories of the tracking points are indicated by red curves superimposed on the M-mode echocardiogram, which is shown in Figure 4. The authors employed the difference between two trajectories obtained by automatic and manual tracking as a measure of tracking accuracy, according to Eqs. (21) and (22).

In Figure 4, it is clear that the derived trajectory using DP with the ME-model method is smoother and exhibits less interactions in the motion tracking curves. The interactions are marked by green cycles. Figure 4 is obtained from two subjects. In the figure, the upper and lower images correspond to the IVS and LVPW, respectively.

Figure 5 shows the results of the evaluation test on the IVS and LVPW using two conventional methods and the proposed method, respectively. Figure 5 (a) and (b) show statistical evaluations of tracking error on the IVS and LVPW. The statistical significance of the proposed method against the two conventional methods, i.e. CWP with the ME-model and Simple Tracking, was assessed with a significance level of $\alpha=0.05$ on both the IVS and LVPW, respectively. 


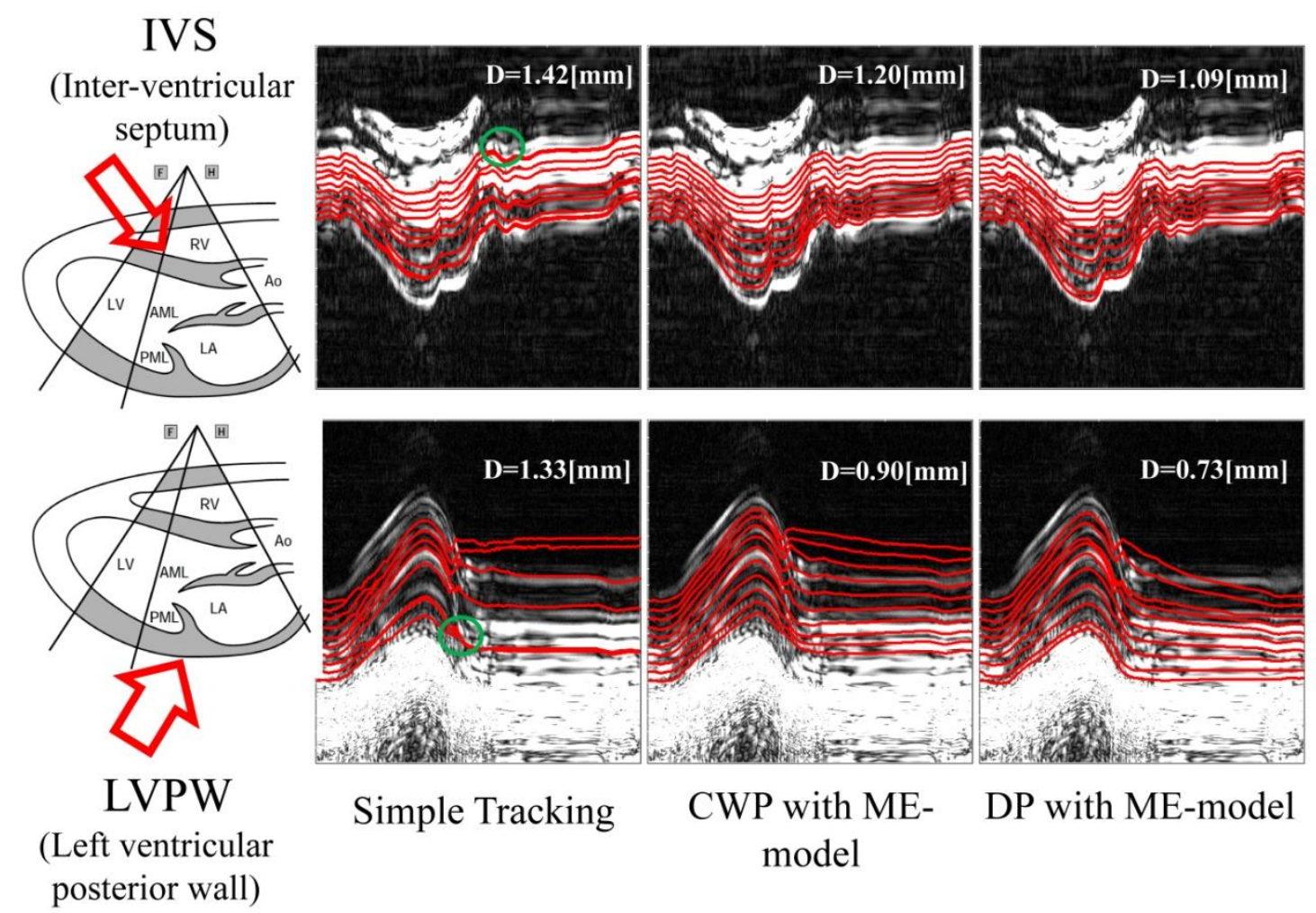

Figure 4: Visual comparison of tracking results: left to right are the tracking results of the Simple Tracking method, CWP with ME-model method [10], and DP with ME-model method, respectively.

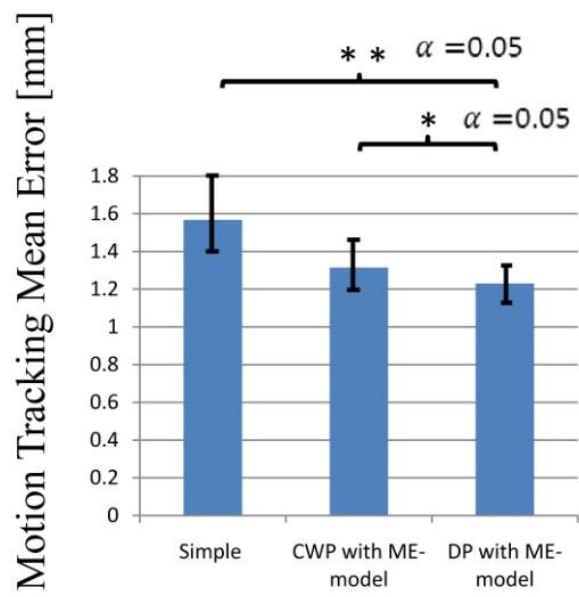

IVS

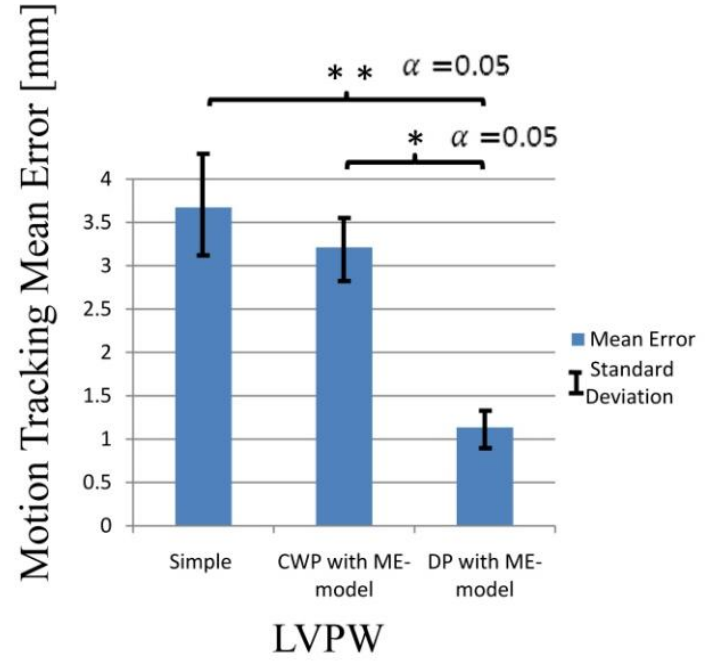

(b)

Figure 5: Statistical evaluation of the tracking error of the tracking methods on (a) the IVS and (b) the LVPW.

This clearly shows that the proposed DP with the ME-model yielded optimal results for both the IVS and LVPW.

Systematic error is also known as regular error in many cases. It expresses the difference between the mean value of a measured attribute and the actual value of the measured attribute in repeatable conditions. Some fixed issues lead to this error in the procedure. It is repeatable, unidirectional and measureable. If the cause can be determined and we can measure systematic error, then systematic error can be reduced or avoided. Systematic error is the main error in quantitative analysis. In this study, a normal fitting is used to estimate the systematic error between manual tracking and automatic tracking using the proposed method. This analysis is applied to the proposed method of validation.

The cardiac cycle includes the systole and diastole periods. In general, the ventricular systole takes $0.3 \mathrm{~s}$ to complete. The movement of the myocardium in systole is much more strenuous than that in diastole. Many cardiovascular diseases can be diagnosed by analyzing the movement of the myocardium in systole. The objective of normal fitting is to judge whether the proposed objective of normal fitting can clinically replace manual tracking by doctors, in order to realize a rapid and high objective diagnosis. 
The difference between manual tracking and automatic tracking at time $\boldsymbol{t}_{k}$ is defined by:

$$
\begin{gathered}
\boldsymbol{S}_{k}=\boldsymbol{m}_{k}-\boldsymbol{a}_{k}, \text { for } \\
k=0,1,2, \ldots, L-1,
\end{gathered}
$$

where $\quad \boldsymbol{m}_{k}=\left(d_{k}^{(0)}, d_{k}^{(2)} d_{k}^{(3)}, \ldots, d_{k}^{(M-1)}\right) \quad$ and $\quad \boldsymbol{a}_{k}=$ $\left(a_{k}^{(0)}, a_{k}^{(2)} a_{k}^{(3)}, \ldots, a_{k}^{(M-1)}\right)$ are tracking points at time $\boldsymbol{t}_{k}$ for manual tracking and automatic tracking, respectively.

Since one cardiac cycle contains two stages, systole and diastole, the normal fitting also contains two stages. Figure 6 (a) and (b) show the results with a normal distribution for the proposed method in diastole and systole, respectively. Here, $\mu$ is the mode, which expresses the distance between the peak of the red line and the origin of the axis: the value of $\mu$ expresses the mean value of the total. The yellow line denotes the mean value of the histogram, and is also the peak point of the fitted wave in the figure. These results show that the proposed method produces only a small difference between systole and diastole. Although the movement in systole is strenuous, the proposed method can still replace the manual tracking method and provide an objective reflection of the movement of the myocardium.

\section{CONCLUSION}

The authors have proposed a dynamic programming technique with a myocardial elastic model technique for deriving motion tracking on the IVS and LVPW in an M-mode echocardiogram. The proposed method employs dynamic programming to guarantee a minimal result. It also increases the robustness against extreme motion tracking error due to speckle noise effects in echocardiograms. According to visual comparisons and statistical evaluation, the experimental results show that this method can effectively alleviate the problems caused by conventional methods. In general, the DP with the ME-model demonstrates superior performance compared to conventional methods in this application.

For future work, the authors wish to point out that there are several other methods and applications that are employed in echocardiograms [13] [14]. The authors will combine the proposed method with such the new modalities in order to improve the quality of diagnoses of cardiovascular diseases.

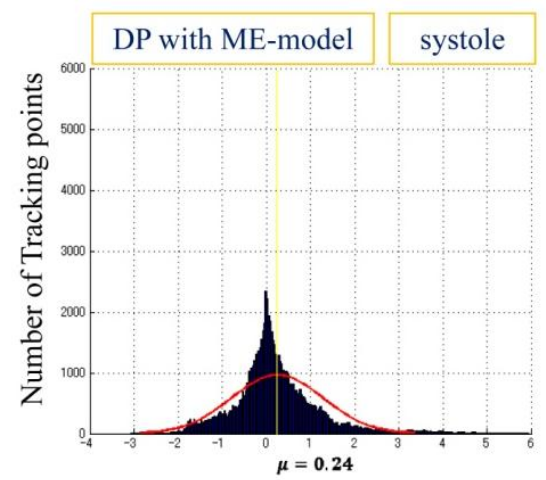

The Error of Motion Tracking $[\mathrm{mm}]$

(a)

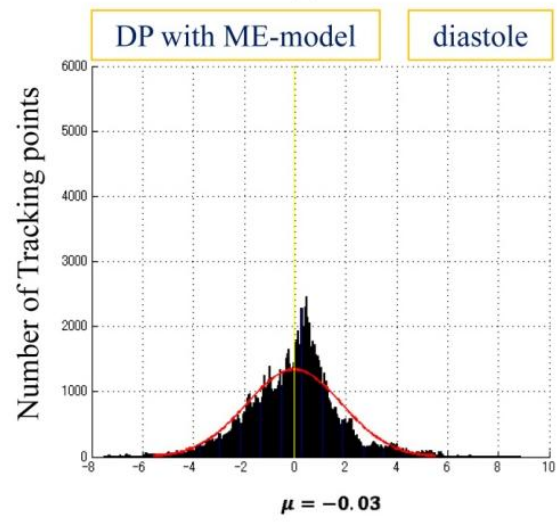

The Error of Motion Tracking[mm]

(b)

Figure 6: Normal fitting for systematic error analysis.

\section{REFERENCES}

[1] Chunke Y, Terada K, Oe S: Motion analysis of echocardiograph using optical flow method. Proc IEEE Int.Conf.Systems, Man, and Cyberpeticsl:672-677,1996

[2] Baraldi P, Sarti A, Lamberti C et al: Evaluation of differential optical flow techniques on synthesized echo images. IEEE Trans Biomedical Engineering 43(3):259272,1996

[3] Suhling M, Arigovindan M, Jansen C et al: Myocardial motion analysis from B-mode echocardiograms. IEEE Trans Image Processing 14(4):525-536,2005

[4] Hokanson DE, Monzersky DJ, Sumner SD et al: A phase-locked echo tracking system for recording arterial diameter changes in vivo. J ApplPhys 32(5):728-733, 1972

[5] Kanai H, Sato M, Koiwa $\mathrm{Y}$ et al: Transcutaneous measurement and spectrum analysis of heart wallvibrations. IEEE Trans Ultrasonics, Ferroelectrics, and Frequency Control 46(5):791-810, 1996

[6] Kanai H, Hasegawa H, Chubachi $\mathrm{N}$ et al: Noninvasive evaluation of local myocardial thickening and its colorcoded imaging. IEEE Trans Ultrasonics, Ferroelectrics, and Frequency Control 44(4):752-768,1997

[7] Kanai H, Koiwa Y, Zhang J: Real-time mesurements of local myocardium motion and arterial wall thickenings. IEEE Trans Ultrasonics, Ferroelectrics, and Frequency Control 46(5):1229-1241,1999 
[8] Tsuruoka S, Kunisada T, Ohyama W et al: Automatic tracking of regional myocardium from ultrasonic rf echo signals using correlation method weighted with confidence. Proc 10th International Conference on Biomedical Engineering: 149-150,2000

[9] Ohyama W, Wakabayashi T, Kimura F et al: Automatic tracking of local myocardial motion by correlation weighted velocity method. Proc. 16th International Conference on Pattern Recognition 1:711-714, 2002

[10] Chao C, Ohyama W, Wakabayashi T, Kimura F, Sekioka, K: Motion Tracking of Local Myocardial Tissue Using a DP Tracking Method on M-mode Echocardiograms. Medical Imaging Technology. Vol. 28, No. 4, pp.271278 (Oct. 2010)

[11] Chao C, Maesako N, Ohyama W, and Wakabayashi T, Sekioka K: Motion Tracking of Local Myocardial Tissue Using DP+E Tracking Method on M-mode Echocardiogram: Fifth International Conference on
Emerging Traends in Engineering\&Technology (Nov,2012 Himeji, Japan)

[12] Chao C, Maesako N, Ohyama W, Wakabayashi T, Kimura F: Tracking of Local Myocardial Motion on MMode Echocardiogram by DP-based Elastic Model" Proceedings of the 14th IASTED International Conference on Signal and Image Processing (SIP 2012),786-031(August2012,Honolulu),

[13] Da-Chuan C, Xiaoyi J: Detection of Arterial Wall in Sonographic Artery Images Using Dual Dynamic Programming. Information Technology in biomedicine, IEEE Transactions in Biomedicine. Vol.16 Issue: 6, pp.792-799, Nov.2008

[14] Aditi R, Shamik S, Jayanta M and Arun K. M: StateBased modeling and Object Extraction From Echocardiogram Video. Information Technology in biomedicine, IEEE Transactions in Biomedicine. Vol. 12, Issue: 3, pp.366-376, May 2008. 